\title{
Theory of intracavity-pumped photorefractive phase-conjugate mirror
}

\author{
R. Yahalom and A. Yariv \\ Thomas J. Watson, Jr. Laboratories of Applied Physics, California Institute of Technology, Pasadena, California \\ 91125
}

Received November 30, 1987; accepted April 7, 1988

\begin{abstract}
We present a new type of phase-conjugate mirror that is based on an externally driven Fabry-Perot interferometer with intracavity-pumped photorefractive material, which is probed by the signal beam. It is shown theoretically that such a configuration leads to multivalued solutions and possibly to bistability. This configuration also permits optical control of the resonator output and electrical control of the phase-conjugate reflectivity.
\end{abstract}

\section{INTRODUCTION}

During the past years there has been considerable research on optical phase conjugation based on photorefractive materials, and many novel devices have been demonstrated. ${ }^{1}$ Photorefractive crystals were shown to have exceptionally high nonlinear gain and were proved to be effective for lowenergy $\mathrm{cw}$ applications. ${ }^{2}$ In this paper we propose to combine these properties with the inherent nonlinear behavior of a Fabry-Perot interferometer.

The use of an externally driven nonlinear cavity with a Kerr-like medium as an optically bistable device has been well documented. ${ }^{3}$ The probing of such a device by a weak signal beam, thereby combining degenerate four-wave mixing (FWM) with the cavity operation, was also investigated. ${ }^{4}$ However, to our knowledge no attention has been given to the behavior of an intracavity photorefractive material when it is probed by an external (not necessarily weak) signal beam that is not part of the cavity.

A typical arrangement for such a (cavity-coupled) phaseconjugate mirror (CC-PCM) is shown in Fig. 1. The pump field $E_{0}$ is coupled into the cavity by a mirror $\left(\mathrm{M}_{1}\right)$ with amplitude reflectivity $r_{1}$. The internal fields are $E_{1}$ (left propagating) and $E_{2}$ (right propagating). The crystal is probed by a signal beam, $E_{4}$, and the phase-conjugate field is $E_{3}$. In this paper we discuss the specific configuration of Fig. 1. Other directions of the $c$ axis and the signal beam are also possible (Fig. 2). Whereas the detailed structure of the results depends on the chosen configuration, the main attributes of a CC-PCM are common to all and will be demonstrated here with the configuration of Fig. 1 only. In this configuration a strong internal field $E_{2}$ is diffracted preferentially into $E_{3}$. $E_{1}$ may thus be of the same order of magnitude as $E_{4}$, giving rise to a relatively large modulation depth of $E_{1} E_{4}{ }^{*}$ and to high phase-conjugate mirror (PCM) reflectivity.

\section{THEORETICAL ANALYSIS AND RESULTS}

In the discussion that follows we assume that all fields are of the same frequency. In such a case the fields $E_{1}$ and $E_{2}$ do not suffer any phase shifts due to the nonlinear interaction, if we assume the usual $\pi / 2$ phase shift between the interference pattern and the induced space-charge field, i.e., a real effective coupling constant $\gamma^{5}$ The notation that we use is similar to that of Ref. 1. For simplicity, we assume that the transmission grating is dominant, that the nonlinear medium is lossless, and that all interacting beams are plane waves. The basic equations are ${ }^{1}$

$$
\begin{aligned}
\frac{\mathrm{d} A_{1}}{\mathrm{~d} z} & =\Gamma A_{4}, \\
\frac{\mathrm{d} A_{4}^{*}}{\mathrm{~d} z} & =-\Gamma A_{1}{ }^{*}, \\
\frac{\mathrm{d} A_{2}{ }^{*}}{\mathrm{~d} z} & =\Gamma A_{3}{ }^{*}, \\
\frac{\mathrm{d} A_{3}}{\mathrm{~d} z} & =-\Gamma A_{2}, \\
\Gamma & =\gamma\left(A_{1} A_{4}{ }^{*}+A_{2}{ }^{*} A_{3}\right) / I_{T}, \\
I_{T} & =\sum_{i=1}^{4} I_{i}, \quad I_{i}=\left|A_{i}\right|^{2},
\end{aligned}
$$

where $\gamma$ is the (in general) complex coupling constant and $A_{i}$ are the field amplitudes.

The most general solution of Eq. (1) is given by

$$
R_{\mathrm{pc}}=4|c|^{2} /(Q / T+\Delta)^{2},
$$

where

$$
\begin{aligned}
& Q=\left(\Delta^{2}+4|c|^{2}\right)^{1 / 2}, \\
& T=-\tanh \left(\gamma l Q / 2 I_{T}\right), \\
& \Delta=I_{2}(l)-I_{1}(0)-I_{4}(0),
\end{aligned}
$$

$R_{\mathrm{pc}}$ is the PCM intensity reflectivity; and $|c|^{2}$ is given by $\left[|c|^{2}-I_{1}(0) I_{2}(l)\right](Q / T+\Delta)^{2}+4|c|^{2}\left[I_{2}(l)+\operatorname{Re}(T) Q /|T|^{2}\right]=0$.

For real $\gamma$ this solution can be manipulated further. We 


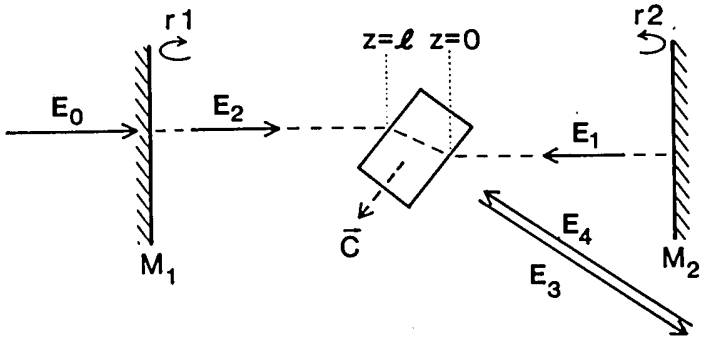

Fig. 1. Intracavity pumped PCM. This specific configuration is the one analyzed in the paper.

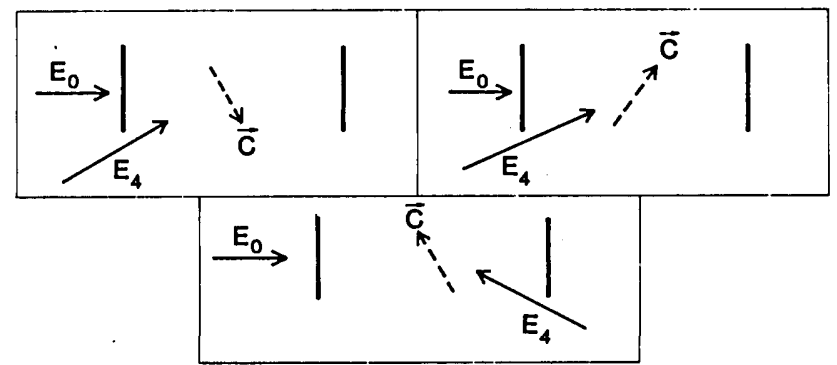

Fig. 2. Examples of three other possible configurations of a CCPCM (in terms of the $c$ axis and probe direction).

normalize all intensities by the probe intensity, so that $I_{4}(0)=1$. It can be shown that Eqs. (2)-(4) lead to the solution

$$
R_{\mathrm{pc}}=\left[I_{1}(0) I_{2}(l)-|c|^{2}\right] /\left[Q / T+I_{2}(l)\right],
$$

where $|c|^{2}$ can now be solved to be

$$
|c|_{1,2}^{2}=\left(\left\{I_{1}(0)\left[I_{2}(l)-R_{\mathrm{pc}}\right]\right\}^{i / 2} \pm\left(R_{\mathrm{pc}}\right)^{1 / 2}\right)^{2} .
$$

Equations (5) and (6) still must be solved numerically in a self-consistent way. $I_{2}(l)$ and $I_{1}(0)$ are the known inputs, and they are given in terms of the boundary conditions

$$
\left|E_{0}\right|^{2} \equiv q=\left[I_{2}(l)+r_{1}{ }^{2}|c|^{2} / I_{2}(l)-2 r_{1}|c| \cos (\theta+\psi)\right] /\left(1-r_{1}{ }^{2}\right) .
$$

Here $\theta$ is the round-trip (geometrical) phase delay, and $\psi$ is the additional phase delay caused by the nonlinear interaction during the round trip through the crystal. Note that for real $\gamma, \psi$ is equal to zero. The second boundary condition is

$$
I_{1}(0)=r_{2}^{2} I_{2}(0) \equiv r_{2}^{2}\left[I_{2}(l)-R_{\mathrm{pc}}\right] \text {. }
$$

We can therefore find that

$$
\begin{aligned}
\Delta & =r_{2}^{2} R_{\mathrm{pc}}+I_{2}(l)\left(1-{r_{2}}^{2}\right)-1, \\
I_{T} & =1+I_{2}(l)\left(1+r_{2}{ }^{2}\right)-r_{2}{ }^{2} R_{\mathrm{pc}} .
\end{aligned}
$$

When Eqs. (7)-(10) are used, the PCM reflectivity [Eqs. (5) and (6)] is determined by the control parameters $\left\{q, \theta, r_{1}, r_{2}\right\}$ only.

The solution described above shows a highly nonlinear dependence of $R_{\mathrm{pc}}$ not only on the input pump-probe ratio $q$ (as expected for the FWM process) but also on the cavity parameters that can be electrically controlled, $(\theta$, as an example, can be piezoelectrically changed.) This dependence also leads, as will be demonstrated, to multivalued solutions even for relatively small coupling $\gamma$.
Two other important outputs are the resonator transmittance $\left(T_{0}\right)$ and reflectance $\left(R_{0}\right)$, which are found to be

$$
\begin{aligned}
& R_{0}=1-\left[I_{2}(l)-|c|^{2} / I_{2}(l)\right] / q, \\
& T_{0}=\left(1-r_{2}^{2}\right)\left[I_{2}(l)-R_{\mathrm{pc}}\right] / q .
\end{aligned}
$$

We see that both $R_{0}$ and $T_{0}$ depend on the PCM reflectivity. It is possible to control the cavity outputs optically by changing $q$, and multivalued solutions are also possible.

In Fig. 3 we show the results $\left(R_{\mathrm{pc}}, R_{0}\right)$ for $r_{1}=0$ and $r_{2}=1$, i.e., for a normal FWM process when one of the pump beams is supplied by the reflection on mirror $\mathrm{M}_{2}$ of the second pump. In Figs. 3(a) and 3(b) we see that for $\gamma l=3$ both $\log \left(R_{\mathrm{pc}}\right)$ and $\log \left(R_{0}\right)$ are almost linear in $\log (q)$ when $I_{0}<$ $I_{4}(0)(q<1)$ and saturate at near unity when $q>1$. This solution is single valued for all values of $q$. In Figs. 3(c) and 3 (d) the case of $\gamma l=6$ is shown. Here a second solution appears for $q>1$, which is due to dominance of the $E_{2}{ }^{*} E_{3}$ grating. 1

These results are to be compared with Fig. 4, in which similar calculations were done for $r_{1}=0.9, r_{2}=1$. In Figs. $4(\mathrm{a})$ and $4(\mathrm{~b})(\gamma l=3)$ we see the appearance of three solutions for $q \leq 2$, for which we have a jump of approximately 2 orders of magnitude in $R_{\mathrm{pc}}$, which brings about the possibility of bistable behavior as a function of $q$. The cavity reflectivity $R_{0}$ is a constant $(\sim 1)$ for the lower branch of $R_{\mathrm{pc}}$ but is increasing as $q$ decreases for the upper branches of $R_{\mathrm{pc}}$. It is, therefore, possible to change the resonator output by controlling the optical input $q$. This behavior can be understood by observing the internal fields. The lower values of $R_{\mathrm{pc}}$ are the results of destructive interference between $E_{1}(l)$ and $E_{0} . \quad\left[E_{1}(l)\right.$ in this case originates mainly from the diffraction of $E_{4}$ but can be relatively large.] As a consequence, $E_{2}(l), E_{2}(0)$, and $E_{3}(0)\left(\equiv R_{\mathrm{pc}}\right)$ are small. In the case of the upper branches of $R_{\mathrm{pc}}$ the opposite is correct. $E_{2}(l)$ is large because of the constructive interference of $E_{0}$ and $E_{1}(l)$. As a result, the term $E_{1} E_{4}{ }^{*}$ leads to a strong grating upon which $E_{2}(l)$ is diffracted to give higher values of the PCM reflectivity.

In Figs. 4(c) and 4(d) we see similar results for $\gamma l=6$. The main differences are a bigger jump (of factor $10^{3}$ ) in $R_{\mathrm{pc}}$ at the bistable point $q \cong 3$ and the appearances of multivalued solutions for $q>1$, as occurred also in the case $r_{1}=0$.

As $\gamma$ is increased, the solutions become richer and more complicated. As an example, we show in Fig. 5 the behavior of $R_{0}$ for $\gamma l=10$.

In all the above examples the cavity was tuned to resonance conditions $(\theta=0)$. When the cavity is slightly detuned, the bistable loop is even clearer. In Fig. 6 we show $R_{\mathrm{pc}}$ for $\gamma l=3$ and $\theta=5^{\circ}$. The corresponding expected hysteresis loop, assuming initial conditions $q=0$, is also displayed.

As we mentioned above, it is also possible to control the PCM reflectivity by changing the cavity round-trip phase. In Fig. $7 \log \left(R_{\mathrm{pc}}\right)$ is shown as a function of $\theta$ for $\gamma l=3$, $\log _{10}(q)=0.2,0.4,0.8$. We observe that for $\log _{10},(q)=0.2$ if initially the system is on the upper branches of $R_{\mathrm{pc}}$ [see also Fig. 4(a)], then, as $\theta$ is increased, self-oscillations cannot contribute substantially to the PCM reflectivity; at $\theta \simeq 40$ the reflectivity drops approximately 3 orders of magnitude. For higher values of $q$ a smooth control of $R_{\mathrm{pc}}$ as a function of $\theta$ is possible. 

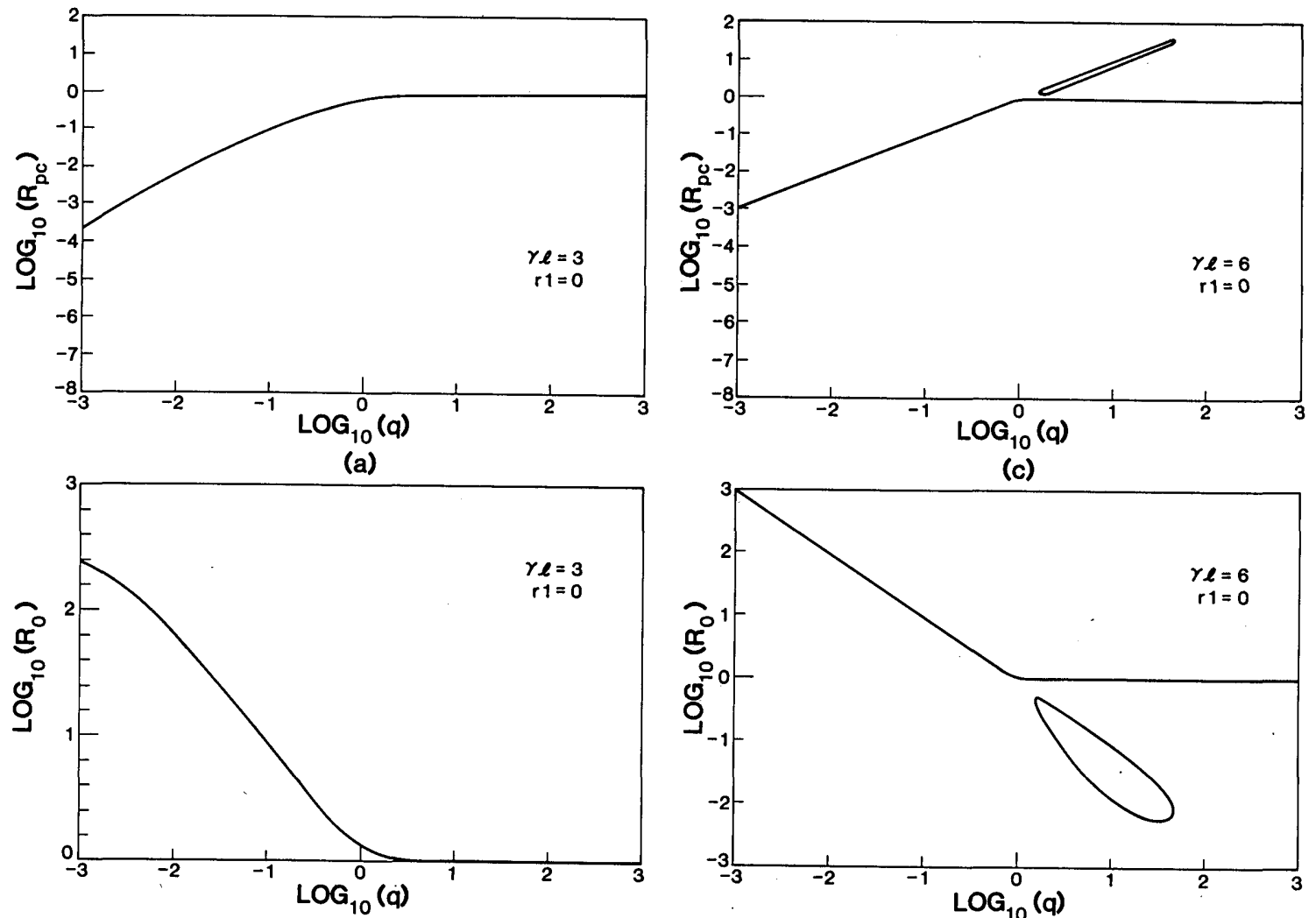

(b)

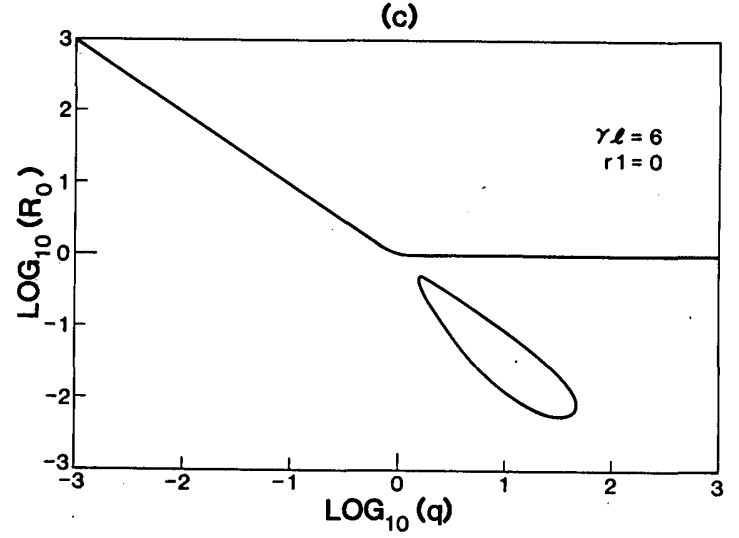

(d)

Fig. 3. Log-log plots of the phase-conjugate reflectivity $R_{\mathrm{pc}}$ and the cavity reflectivity $R_{0}$ as a function of the pump-probe ratio $q$ for the case of an open cavity $\left(r_{1}=0\right)$.
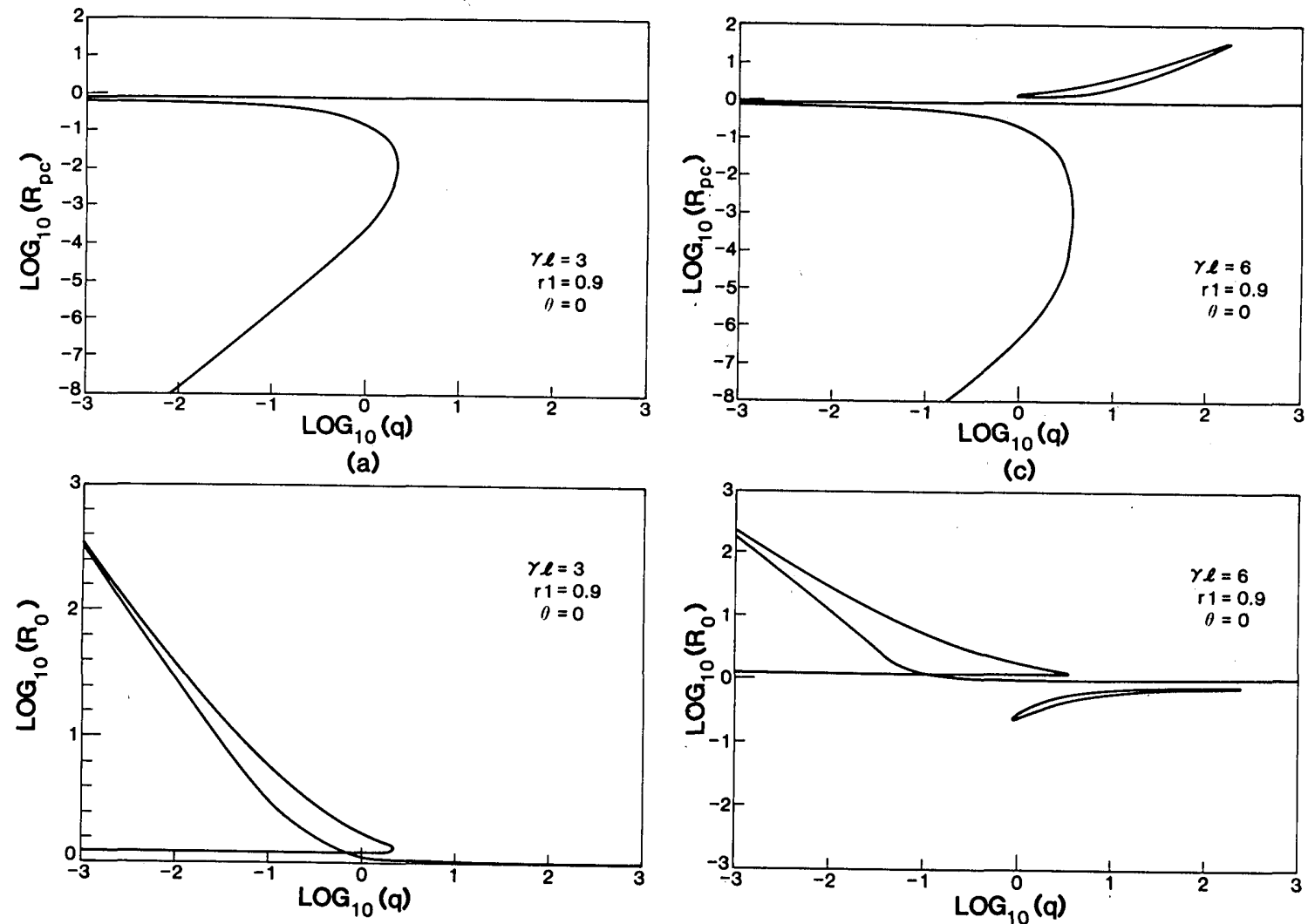

(c)

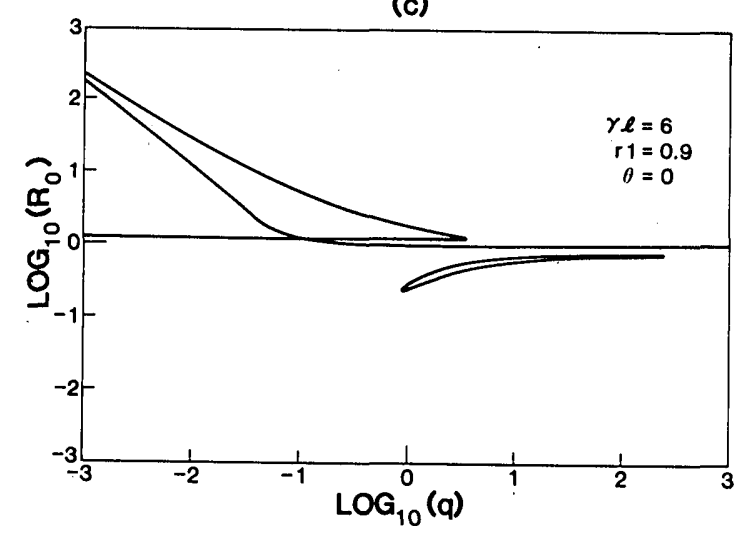

(b)

(d)

Fig. 4. $R_{\mathrm{pc}}$ and $R_{0}$ as a function of $q$ for the case $r_{1}=0.9$. 


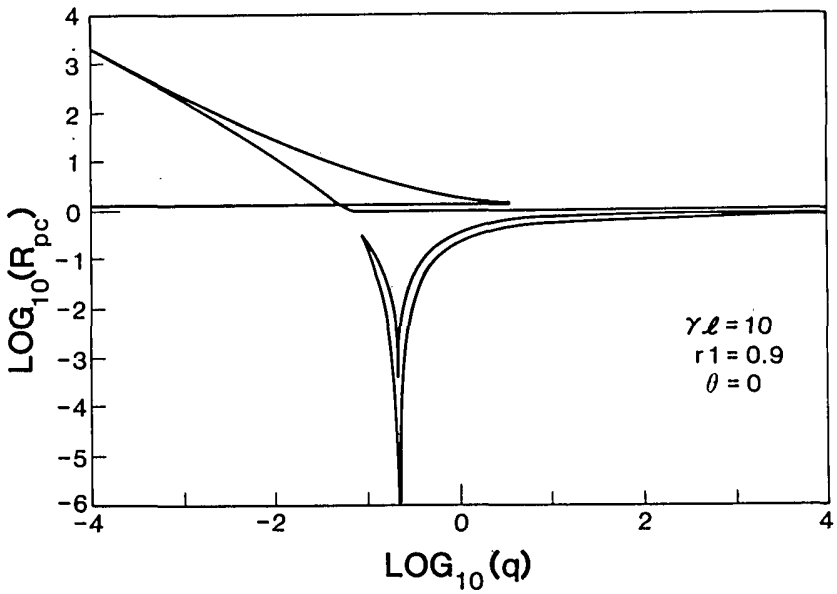

Fig. 5. A plot of the cavity reflectivity $R_{0}$ as a function of the input parameter $q$ for a high-gain $(\gamma l=10)$ crystal.

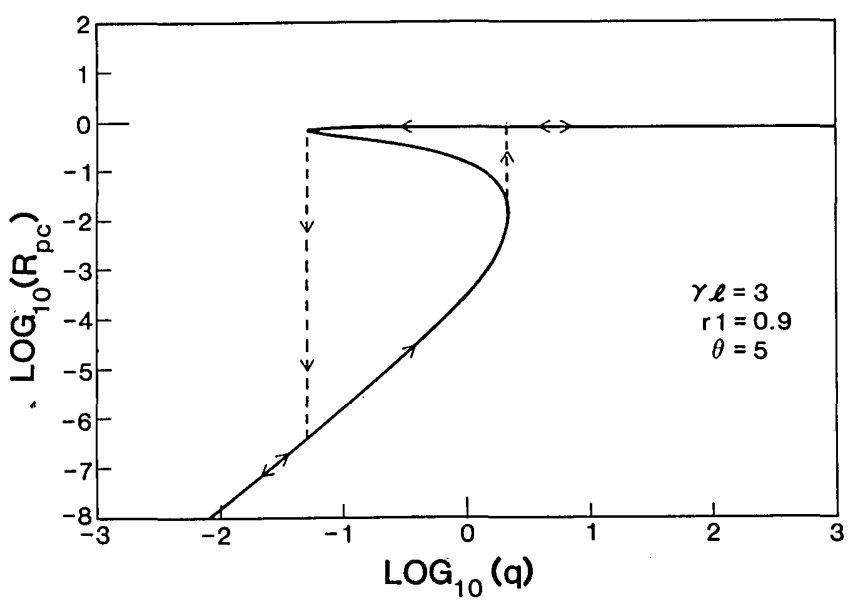

Fig. 6. $R_{\mathrm{pc}}$ and $R_{0}$ as a function of $q$ for a detuned resonator: $\gamma l=$ $3, \theta=5^{\circ}$.

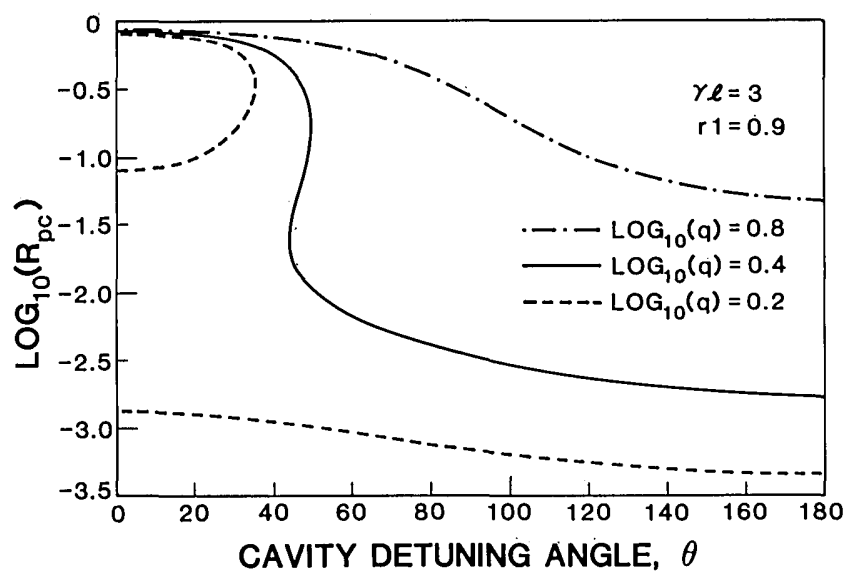

Fig. 7. The dependence of $R_{\mathrm{pc}}$ on the cavity detuning angle $\theta$, for $\gamma l$ $=3$ and three values of the pump-probe ratio $q$.

In the above calculations we did not include the possible appearance of detuned oscillations inside the cavity when $\theta$ $\neq 2 \pi m$, which may be important for $q<1$ even when the external pump and probe fields are of equal frequencies. Such oscillations are known to happen in the absence of external pumping ( $q=0$, i.e., a detuned linear self-pumped
$\mathrm{PCM}^{5}$ ). It is impossible to predict from the steady-state analysis how much of the incoming probe field $E_{4}$ participates in the usual degenerate FWM process (thus creating a fixed grating with the pump field) and what part of $E_{4}$ is channeled into detuned self-oscillations (creating a moving grating). A detailed dynamic investigation is needed. We note, however, that for a high-finesse resonator, detuned oscillations are limited only to a small cavity detuning angle, ${ }^{5}$ beyond which they cannot be sustained. The main features of the dependence of $R_{\mathrm{pc}}$ on $\theta$ for small values of $q$ ( $q$ $\leq 1)$ can therefore also be found from our previous analysis.

Another means for controlling the phase-conjugate reflectivity and the cavity behavior is detuning of the probe frequency relative to the pump frequency. Because of the frequency offset between the pump and the signals beams, the light interference grating moves in space. Because of the finite response time $(\tau)$ of the grating in the photorefractive material, a phase lag is induced between the index grating and the interference grating; the coupling constant becomes complex and is given by

$$
\gamma=\gamma_{0} /(1+i \delta \tau)
$$

where $\gamma_{0}$ is the coupling constant with zero detuning and $\delta$ is the detuning frequency.

To see the effect of complex $\gamma$, we follow the procedure of Ref. 5 and find that for our boundary conditions, with $I_{4}(0)$ $=1$, the round-trip phase is given by

$$
\psi=-\int_{0}^{l} \operatorname{Im}\left\{\frac{\gamma I_{4}\left(1+f+I_{34}+I_{34} / f\right)}{\left[2 I_{2}(l)-\Delta\right]}\right\} \mathrm{d} z,
$$

where

$$
\begin{aligned}
f & =\frac{-2\left[2|c|^{2}+(Q / \tilde{T}+\Delta) I_{2}(l)\right]}{(Q / \tilde{T}+\Delta)\left[Q / \tilde{T}+2 I_{2}(l)-\Delta\right]}, \\
I_{4} & =\frac{I_{2}(l)\left(1-I_{12}\right)-\Delta}{1-I_{12} I_{34}}, \\
I_{12} & =\frac{\left|\left[Q / \tilde{T}+2 I_{2}(l)-\Delta\right]\right|^{2}|c|^{2}}{\left|\left[2|c|^{2}+(Q / \tilde{T}+\Delta) I_{2}(l)\right]\right|^{2}}, \\
I_{34} & =4|c|^{2} /|(Q / \tilde{T}+\Delta)|^{2} .
\end{aligned}
$$

Here, $\tilde{T}=\tanh \left\{\gamma Q(l-z) / 2\left[2 I_{2}(l)-\Delta\right]\right\}$ and $Q=\left(\Delta^{2}+\right.$ $\left.4|c|^{2}\right)^{1 / 2}$. $\quad|c|$ and $\Delta$ are given by Eqs. (4) and (9), respectively.

From Eq. (14) we see that the dependence of $I_{2}(l)$ (and, therefore, of $R_{\mathrm{pc}}$ ) on the boundary condition (i.e., on the pump-probe ratio $q$ ) becomes even more involved, as it is now a function of $\psi\left[I_{2}(l)\right]$ [note that $I_{2}(l)$ also depends on $\delta$ ]:

$q=\left(I_{2}(l)+r_{1}{ }^{2}|c|^{2} / I_{2}(l)-2 r_{1}|c| \cos \left\{\psi\left[I_{2}(l)\right]\right\}\right) /\left(1-r_{1}{ }^{2}\right)$.

When Eq. (19) is combined with Eqs. (2)-(4), (9), (10), and (13)-(18), the explicit solutions for $R_{\mathrm{pc}}$ can be found.

\section{CONCLUSION}

We have suggested and explored the theory of a CC-PCM when the crystal is intracavity pumped. We have shown that this system can have bistability and multivalued solutions as a function of the pump-probe ratio $q$ and detuning $\delta$, even for smaller nonlinear gain $\gamma$. When $q$ is changed, the 
cavity output can be optically controlled and the PCM reflectivity can be controlled externally by varying the cavity parameters $(\theta)$ electrically.

\section{ACKNOWLEDGMENTS}

This research was supported by the U.S. Air Force Office of Scientific Research and by the U.S. Army Research Office.

\section{REFERENCES}

1. M. Cronin-Golomb, B. Fischer, J. O. White, and A. Yariv, IEEE J. Quantum Electron. QE-20, 12 (1984).

2. For a review, see T. J. Hall, R. Jaura, L. M. Connors, and P. D. Foote, Prog. Quantum Electron. 10, 77 (1985).

3. H. M. Gibbs, Optical Bistability (Academic, New York, 1985).

4. G. P. Agrawal, J. Opt. Soc. Am. 73, 654 (1983).

5. S. K. Kwong, M. Cronin-Golomb, and A. Yariv, IEEE J. Quantum Electron. QE-22, 1508 (1986). 